\title{
Drug pipeline: Q410
}

\section{Wayne Peng}

The first recombinant human protein made in transgenic rabbit milk was approved in October by the European Medicines Agency. Proofof-efficacy data in human phase 2 studies were obtained for several nucleic acid therapies: OncoGenex Pharmaceuticals' custirsen and

\section{Top ten disease grouped by pipeline size}

Oncology drugs dominate the development pipeline.

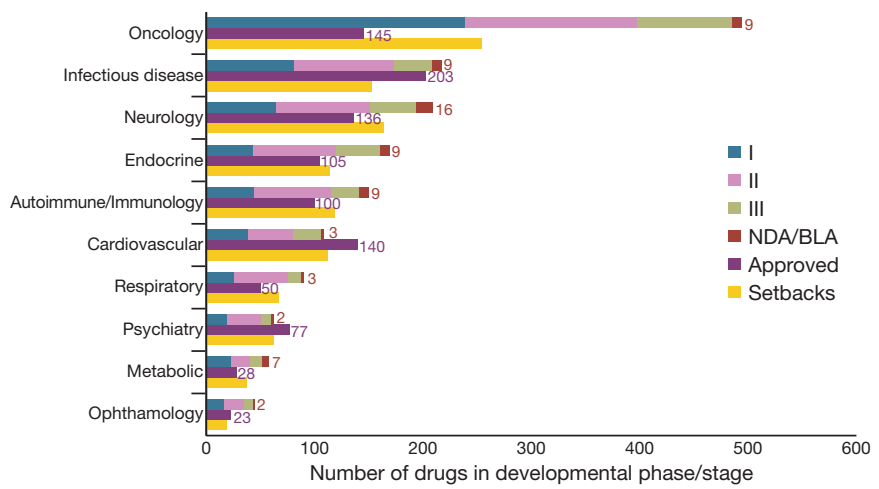

Source: BioMedTracker, a service of Sagient Research

Notable trial results (September-December 2010)

\begin{tabular}{llll}
\hline Drug name & Company & Indication & Result summary \\
\hline Teleprevir & Vertex & Hepatitis C & Final phase 2a trial results showed greater \\
& Pharmaceuticals & $\begin{array}{l}\text { virus (HCV) } \\
\text { infection }\end{array}$ & $\begin{array}{l}\text { anti-HCV activity in combination therapy } \\
\text { with peg-interferon and ribavirin (Amer. } \\
\text { Assoc. Study Liver Dis., Abstract 828, } \\
\end{array}$ \\
& & 2010).
\end{tabular}

Boceprevir Merck/Schering- HCV Final phase 3 trial results met primary Plough infection endpoint in combination therapy with peginterferon and ribavirin (Amer. Assoc. Study Liver Dis., Abstract 216, 2010).

Iniparib Sanofi-aventis/ Breast Phase 2b study met primary endpoint, BiPar Sciences cancer safety and tolerability, and showed significant clinical benefit for the poly ADP-ribose polymerase inhibitor (N. Engl. J. Med. doi: 10.1056/NEJMoa1011418, 2011). Phase 3 trial results for the fully human anti-IL-12/IL-23 mAb met two co-primary endpoints compared to placebo and Enbrel (European Academy of Dermatology and Venereology, 10/11/2010).

Custirsen OncoGenex Prostate Phase 2 trial data of the clusterin-inhibiting Pharmaceuticals cancer antisense oligonucleotide met primary endpoint and showed significant survival benefit with docetaxel combined therapy (J. Clin. Oncol. 28, 4247-4254, 2010).

Trabedersen Antisense Pharma Brain cancer Phase $2 \mathrm{~b}$ trial for the phosphorothioate transforming growth factor beta 2 -specific antisense oligodeoxynucleotide failed to meet primary endpoint but showed clinically relevant benefit (Neuro. Oncol. doi:10.1093/neuonc/noq142, 2010). VX-770 Vertex Cystic Phase 2 trial demonstrated safety of the Pharmaceuticals fibrosis small-molecule potentiator of the cystic fibrosis transmembrane conductance regulator and reached statistical significance for a secondary endpoint, sweat chloride concentration (N. Engl. J. Med. 363 1991-2003, 2010).

Ampligen Hemispherx Chronic Phase 3 trial of the experimental double(rintatolimod) Biopharma fatigue stranded RNA drug reduced the chance of syndrome long QT syndrome and alleviated medication dependence compared with placebo (J. Applied Res. 10, 80-87, 2010).

Source: BioMedTracker, a service of Sagient Research (http://www. biomedtracker.com/). $\mathrm{mAb}$, monoclonal antibody.
Hemispherx Biopharma's Ampligen (rintatolimod) met their endpoints, whereas Antisense Pharma's trabedersen showed only a clinical benefit. Elsewhere, results were positive for Vertex Pharmaceuticals' VX-770 in a phase 2 trial of cystic fibrosis patients.

\begin{tabular}{|c|c|c|c|}
\hline Drug name & Indication & Approval & Drug information \\
\hline $\begin{array}{l}\text { Gilenya } \\
\text { (fingolimod) }\end{array}$ & $\begin{array}{l}\text { Multiple } \\
\text { sclerosis }\end{array}$ & $9 / 21 / 10$ FDA & $\begin{array}{l}\text { Novartis' sphingosine 1-phosphate } \\
\text { receptor agonist }\end{array}$ \\
\hline Ruconest (EU)/ & Hereditary & 10/28/10 EMA; & ; Pharming's recombinant human $\mathrm{C} 1$ \\
\hline $\begin{array}{l}\text { Rhucin (US) } \\
\text { (conestat alfa) }\end{array}$ & angioedema & $\begin{array}{l}\text { PDUFA date } \\
10 / 28 / 11\end{array}$ & $\begin{array}{l}\text { elastase inhibitor produced from } \\
\text { transgenic rabbit milk }\end{array}$ \\
\hline $\begin{array}{l}\text { Egrifta } \\
\text { (tesamorelin) }\end{array}$ & HIV lipodystrophy & $11 / 15 / 10$ FDA & $\begin{array}{l}\text { Theratechonologies' peptide frag } \\
\text { ment of human growth hormone } \\
\text { releasing factor }\end{array}$ \\
\hline
\end{tabular}

Notable regulatory setbacks (September-December 2010)

\begin{tabular}{|c|c|c|c|}
\hline Drug name & Company & Indication & Setback summary \\
\hline $\begin{array}{l}\text { Bydureon } \\
\text { (exenatide LAR) }\end{array}$ & $\begin{array}{l}\text { Amylin } \\
\text { Pharmaceuticals }\end{array}$ & $\begin{array}{l}\text { Type } 2 \\
\text { diabetes }\end{array}$ & $\begin{array}{l}\text { 10/19/10 FDA issued a complete } \\
\text { response letter requesting additional } \\
\text { studies, delaying potential approval until } \\
2011 \text {. }\end{array}$ \\
\hline $\begin{array}{l}\text { Numax } \\
\text { (motavizumab) }\end{array}$ & $\begin{array}{l}\text { Medlmmune- } \\
\text { AstraZeneca }\end{array}$ & $\begin{array}{l}\text { Respiratory } \\
\text { syncytial } \\
\text { virus }\end{array}$ & $\begin{array}{l}12 / 21 / 10 \text { AstraZeneca withdrew BLA for } \\
\text { the humanized monoclonal antibody. }\end{array}$ \\
\hline $\begin{array}{l}\text { Zalbin (albinter- } \\
\text { feron alfa-2b; } \\
\text { also known as } \\
\text { Albuferon or }\end{array}$ & $\begin{array}{l}\text { Human Genome } \\
\text { Sciences/Novartis }\end{array}$ & Hepatitis C & $\begin{array}{l}10 / 4 / 2010 \text { FDA issued a complete } \\
\text { response letter rejecting the BLA. } \\
\text { European MMA was withdrawn earlier } \\
\text { on } 4 / 19 / 10 \text {. }\end{array}$ \\
\hline
\end{tabular}

Albuferon or

Joulferon)

Simplirix GlaxoSmithKline Herpes 9/30/10 Development for vaccine comsimplex virus prising HSV glycoprotein subunit gD2 (HSV) and adjuvant SBAS4/ASO4 stopped after infection missing primary endpoint of phase 3 trial.

Vismodegib Genentech/Roche Ovarian 10/11/10 Development of Smoothened cancer antagonist stopped after missing primary endpoint in phase 2 trial.

ISIS 369645 Isis Asthma 11/4/10 Company discontinued development of antisense drug due to insufficient improvement in phase $2 \mathrm{a}$ trial.

TroVax Oxford Biomedica Renal cell 10/14/10 Phase 3 trial for vaccine cancer comprising tumor antigen 5T4 failed to meet overall survival primary endpoint (Clin. Cancer Res. doi:10.1158/10780432, 2010).

Source: BioMedTracker, a service of Sagient Research (http://www.biomedtracker.com/). BLA, Biologic License Application; LAR, long-acting release; MMA, marketing authorization application.

Notable upcoming decisions (Q1 2011)

Drug Name

Company Indication Expected approval decision

Exocrine 1/31/10 PDUFA. Oral recombinant

(liprotamase) pancreatic enzyme replacement therapy that con-

Idiopathic 2/1/11-3/31/11 MAA decision date

fibrosis p38 kinase inhibitor demonstrated

dosage-dependent response and safety. European Respiratory Society, 9/19/10 Abstract 388.

Uplyso Protalix Gaucher 2/25/11 PDUFA date. Recombinant

(taliglucerase BioTherapeutics/ disease human glucocerebrosidase demon-

alfa) Pfizer strated both safety and effectiveness, according to company press release on $11 / 2 / 10$.

Other expected approvals in Q1 delayed from the previous quarter include: Human Genome Sciences' Benylsta (belimumab), Madarex/Bristol-Meyers Squibb's Yervoy (ipilimumab), MannKind's Afrezza (inhaled insulin) and LG Life Sciences' LB03002 (RS-rHGH).

See Nat. Biotechnol. 28, 998, 2010 for details.

Source: BioMedTracker, a service of Sagient Research (http://www.biomedtracker.com/). PDUFA, Prescription Drug User Fee Act. MMA, market authorization application.

Wayne Peng is Emerging Technology Analyst, Nature Publishing Group 